\title{
Evaluating the Failures of Data Centers in Cloud Computing
}

\author{
Preeti Gupta \\ CSE Deptt. \\ MIET, Jammu
}

\author{
Chaahat Gupta \\ CSE, Deptt. \\ MIET, Jammu
}

\begin{abstract}
This letter presents a study on the various features of the cloud computing its models and services. Cloud computing has become the newest concern in IT world to provide various services via internet computing. Thus cloud computing is based on the internet based computing where indispensable collective servers offers software, infrastructure, platform, storage and other resources and presents to customers on a pay-as-you-use foundation. The overall objective of this work is to evaluate the various failures of the cloud data centers. To achieve the objectives a review has been conducted on the various research papers and the various failures has been presented.
\end{abstract}

\section{Index Terms}

\section{CLOUDS, PAY AS YOU GO, XaaS, ON-DEMAND.}

\section{INTRODUCTION}

Cloud computing is one of the fieriest, growing technology in the IT world. Cloud computing [1], [2], [3] is currently emerging as a powerful way to transform the IT industry to build and deploy custom applications. The word cloud is similar to the Internet. But in real cloud computing model, it means to have all the software and data held on a server or a group of servers, and to access them through the internet. In certain cases the client is a device furnished with a nominal OS and running a web browser. Users have facility to use these services existing on the cloud without the knowledge of managing of the resources is done. Thus the users can only focus on their processing rather than wasting time and attaining knowledge on the resources needed to cope up their processes [2]. To view cloud computing is to believe of the email account. To access the account, open the browser, go to the email client and simply log-in and the important part is having the Internet access. The email account is not housed i.e. the whole of the Yahoo or Gmail software is not installed on the computer instead it is accessed by the Internet connection [2].

"Cloud" is the aggregation of Servers, Low end computers and storage hosting the program and data, Accessed via Internet anywhere from world [4] [5] [6].

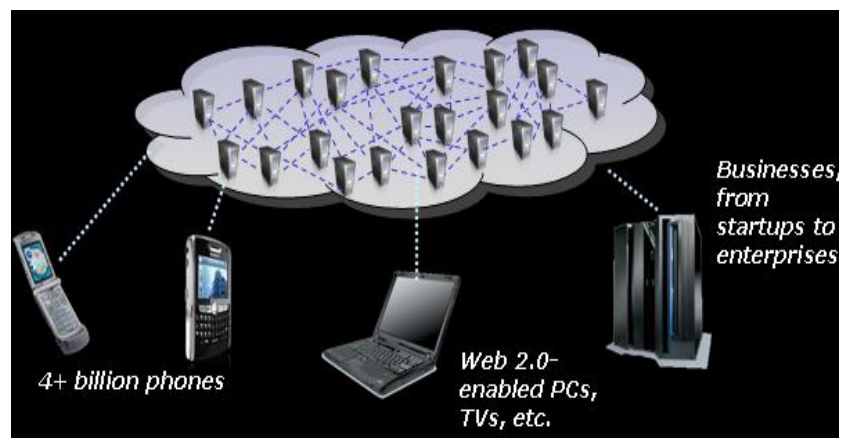

Figure 1: Cloud computing

\subsection{Cloud Computing Characteristics}

\subsubsection{On-Demand}

A fundamental thought of the cloud computing is to convey the resources at whatever point there is need. From the client's perspective the accessible registering resources are almost boundless i.e., the client does not think about the set of servers placed at one site therefore it is the obligation of the distributed computing supplier to have satisfactory resources to satisfy the solicitations of all their clients. Utilizing processing resources on-interest is a standout amongst the most favored capabilities for a substantial number of endeavors on the grounds that it uproots the requirement for arranging forward, getting, and settling the resources they may require sooner or later. This permits the client to forestall making an unnecessary financing in servers.

\subsubsection{Pay-per-Use}

In case of cloud computing, the person pays only for the usage whereas in the case of the traditional computing there is a need to locate the resources physically on the side of the customer. The cloud computing provides the ability of paying the service provider depending only on the use of the customer.

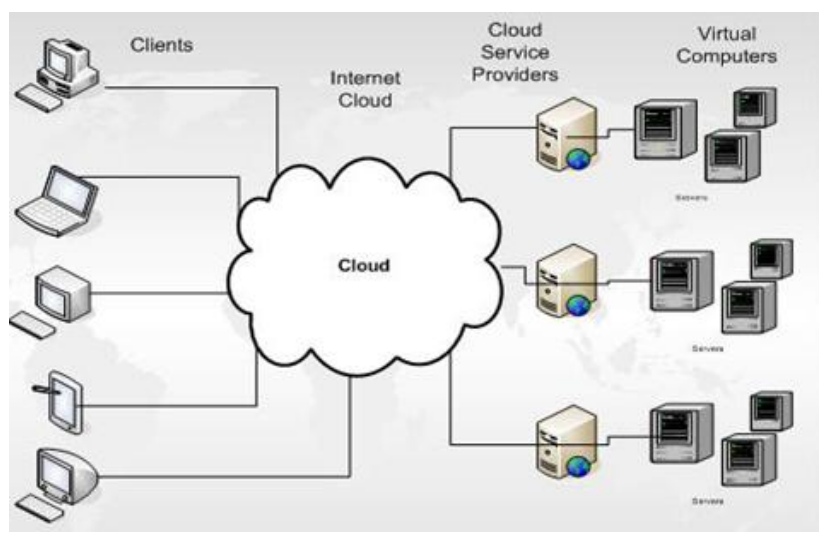

Figure 2: Cloud computing working

\subsubsection{Rapid Elasticity}

The cloud supplier scales up or down the resources relying on the particular of an administration level understanding that are accommodated the changing client needs. This administration characterizes the time for the cloud supplier to reaction back. Such an agreement is needed by the cloud supplier, on the grounds that the cloud supplier does not indeed have innumerable resources, so relying on the administration level understanding the supplier need to run across a set of disseminations of resources that satisfy the requests of their clients generally the administration level assention oblige a punishment that the supplier need to pay to every client for not meeting the understanding. 


\subsubsection{Maintenance and Upgrading}

The supplier keeps up the figuring resource instead of the client. In this way the supplier jelly and overhauls the resource. Subsequently all change of the resource are avoided the client's perspective, however this may be genuine in the perfect case. Now and again because of a portion of the reasons the client is moved from one stage to the next, all things considered the client may be furnished with any resource.

\subsection{Types of Cloud Computing}

\subsubsection{Private Cloud}

The cloud foundation has been arranged, and is protected and worked for a particular association. They are utilized by that gathering. An immaculate private cloud is assembled for the private utilization of one client, who keeps and completely controls this cloud. The recognizing peculiarity of any private cloud is that the way that the cloud is utilized by a particular client. A private cloud may be kept up by the customer, yet fabricated, altered, and fulfilled by an outsider instead of the customer. The physical servers may be arranged at the client's destinations. The substitute to a private cloud is a 'virtual private cloud'. In such a virtual private cloud a client is appointed a private cloud inside the physical setup of an open cloud. Because of the imparting of positive assets inside the cloud the client could be ensured that their information put away on and handling is carried out just on given servers.

\subsubsection{Public Cloud}

The cloud framework is open to the general population by a cloud administration supplier with the Internet association. This permits a client to utilize an administration as a part of the cloud with pay-as-you-go premise as opposed to acquiring the entire cloud.

\subsubsection{Community Cloud}

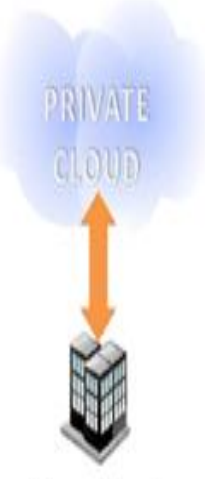

Private Cloud

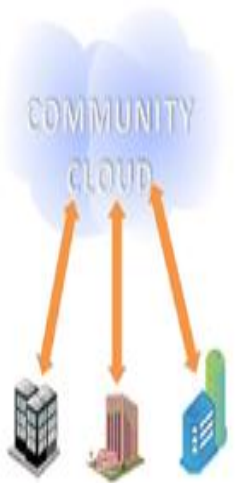

Community Cloud

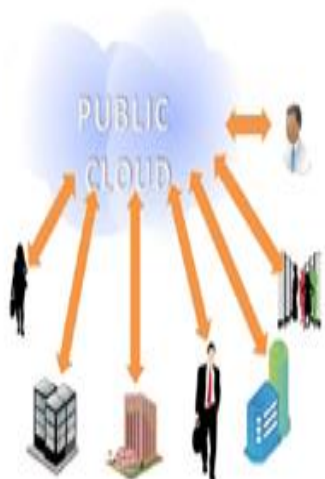

Public Cloud
Figure 3: Deployment Models

The cloud infrastructure is shared among a number of organizations with like interests and needs. This help in decreasing the capital expenditure costs as the expenses are united among the organizations.

\subsubsection{Hybrid Cloud}

The cloud base includes various billows of any sort, however the mists have the capacity through their interfaces to be exchanged starting with one cloud then onto the next. This might be a mix of private and open mists that backing the necessity to hold some information in an association, furthermore the need to offer offices in the cloud.

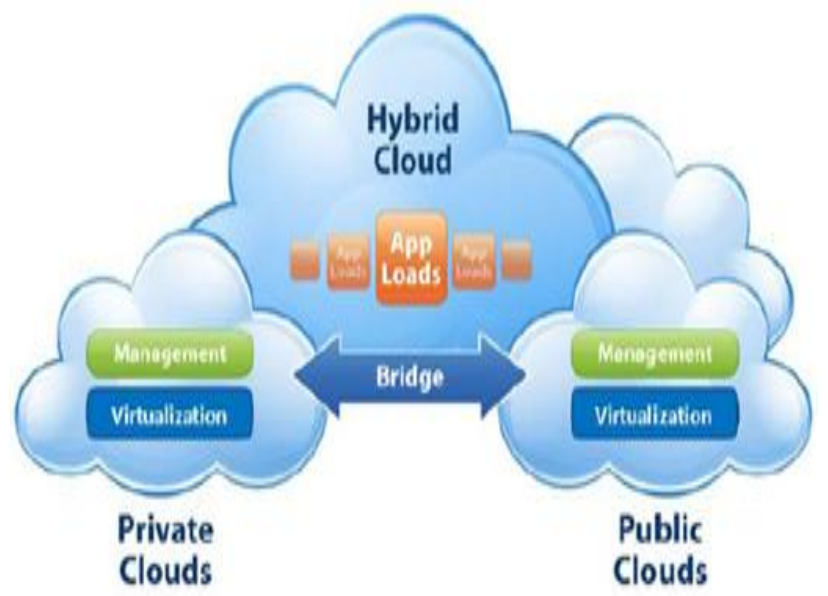

Figure 4: Hybrid Cloud
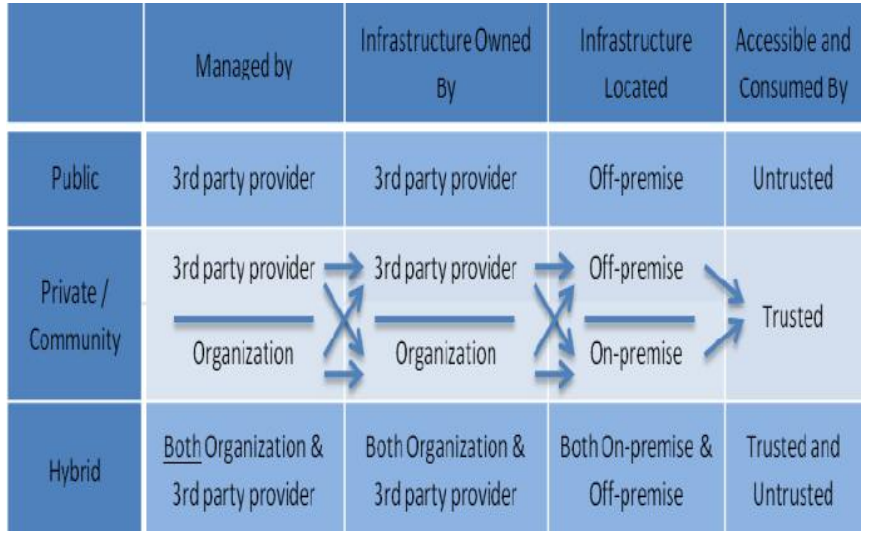

Figure 5 - Difference between Ddeployment models

\section{CLOUD COMPUTING SERVICE MODELS}

Each service provider provides a exact function allowing user to have less or more control on the cloud depending on the type i.e. private, public, hybrid or community. The cloud need vary depends on how the cloud will be used i.e. the space and the resources related with the cloud. Cloud computing can be classified by the model of service it offers into many different groups. These are defined using the XaaS taxonomy, where "X" can be Software, Platform, or Infrastructure, and the final "S" is for Service.

In addition to above some professionals list the following building blocks of cloud computing:

1. Storage-as-a-Service

2. Database-as-a-Service

3. Information-as-a-Service

4. Process-as-a-Service

5. Application-as-a-Service

6. Integration-as-a-Service

7. Security-as-a-Service

8. Management/Governance-as-a-Service

9. Testing-as-a-Service 


\subsection{Models}

This section contains the various well-known models of the cloud computing.

\subsubsection{IaaS (Infrastructure as a Service)}

The capability provided to the customer of IaaS is raw storage space, computing, or network resources with which the customer can run and execute an operating system, applications, or any software that they choose. The cloud customer is not able to control the distribution of the software to a specific hardware platform or change parameters of the underlying infrastructure, but the customer can manage the software deployed (generally from the boot level upward)[7].

\subsubsection{PaaS (Platform as a Service)}

In the case of PaaS, the cloud provider not only provides the hardware, but they also provide a toolkit and a number of supported programming languages to build higher level services (i.e. software applications that are made available as part of a specific platform). The users of PaaS are typically software developers who host their applications on the platform and provide these applications to the end-users.[8]

\subsubsection{SaaS (Software as a Service)}

The SaaS customer is an end-user of complete applications running on a cloud infrastructure and offered on a platform on-demand. The applications are typically accessible through a thin client interface, such as a web browser. The customer does not control either the underlying infrastructure or platform, other than application parameters for specific user settings[7] [9].

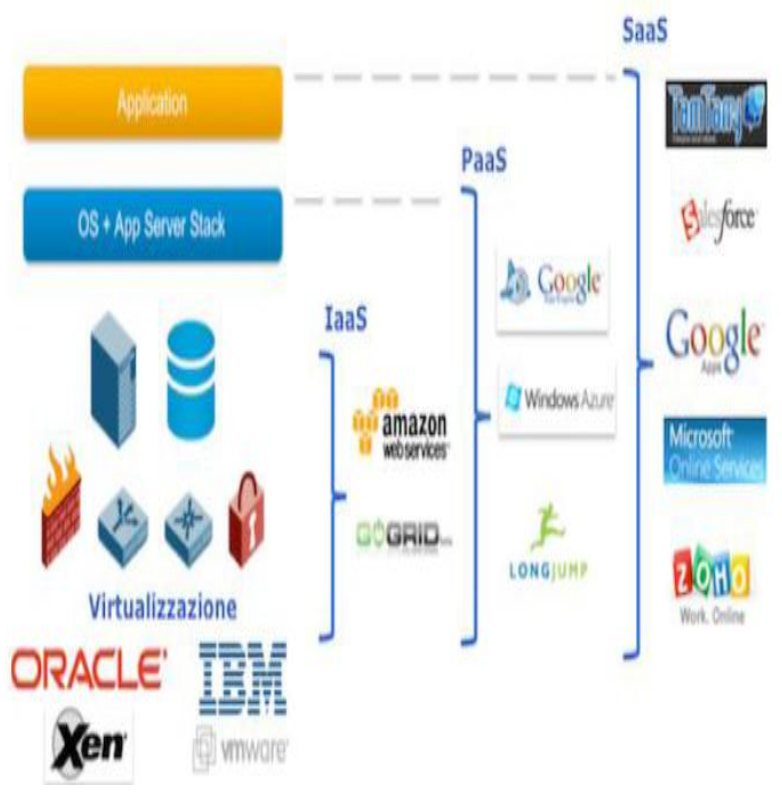

Figure 5 - Cloud computing service models

\subsubsection{Deployment Models}

Clouds can also be classified based upon the underlying infrastructure deployment model as Public, Private, Community, or Hybrid clouds. The different infrastructure deployment models are distinguishing by their architecture, the location of the datacenter where the cloud is realized, and the needs of the cloud provider's customers (for example, due to regulatory, legal, or other requirements).

\section{FAILURES IN CLOUD COMPUTING 3.1 Failure of Monocultures}

The uptime of Cloud Computing based solution is an advantage, when compared to businesses running their own infrastructure, but often overlooked is the co-occurrence of downtime in vendor-driven monocultures. The use of globally decentralized data centres for vendor Clouds minimises failure, aiding its adoption. However, when a cloud fails, there is a cascade effect crippling all organisations dependent on that Cloud, and all those dependent upon them. This was illustrated by the Amazon (S3) Cloud outage [10], which disabled several other dependent businesses. So, failures are now system-wide, instead of being partial or localised. Therefore, the efficiencies gained from centralizing infrastructure for Cloud Computing are increasingly at the expense of the Internet's resilience.

\subsection{Convenience vs Control}

The growing popularity of Cloud Computing comes from its convenience, but also brings vendor control, an issue of everincreasing concern. For example, Google Apps for in-house email typically provides higher uptime [11], but its failure [12] highlights the issue of lock-in that comes from depending on vendor Clouds. The even greater concern is the loss of information privacy, with vendors having full access to the resources stored on their Clouds. So much so the British government is considering a ' $\mathrm{G}$ Cloud' for government business applications [13]. In especially touchy instances of SMEs and new companies, the supplier purchaser relationship that Cloud Computing encourages between the holders of assets and their clients could conceivably be inconvenient, as there is a potential clash of enthusiasm for the suppliers. They benefit by giving resources to cutting-edge players, additionally wish to keep up predominant positions in their customer confronting commercial ventures.

\subsection{Environmental Impact}

The other major concern is the ever-increasing carbon footprint from the exponential growth [14] of the data centres required for Cloud Computing. With the industry expected to exceed the airline industry by 2020 [15], raising sustainability concerns [16]. The industry is being motivated to address the problem by legislation [16], [17], the operational limit of power grids (being unable to power anymore servers in their data centres) [18], and the potential financial benefits of increased efficiency [19], [15]. Their primary solution is the use of virtualisation4 to maximise resource utilization [20], but the problem remains [21], [22].

While these issues are endemic to Cloud Computing, they are not flaws in the Cloud conceptualisation, but the vendor provision and implementation of Clouds [23], [24], [25]. There are attempts to address some of these concerns, such as a portability layer between vendor Clouds to avoid lock-in [26]. However, this will not alleviate issues such as interCloud latency [27]. An open source implementation of the Amazon (EC2) Cloud called Eucalyptus [28], allows a data centre to execute code compatible with Amazon's Cloud. Allowing for the creation of private internal Clouds, avoiding vendor lock-in and providing information privacy, but only for those with their own data centre and so is not really Cloud Computing (which by definition is to avoid owning data centres [29]). Therefore, vendor Clouds remain synonymous with Cloud Computing [29]. Our response is an alternative model for the Cloud conceptualisation, created by combining the Cloud with paradigms from Grid Computing, principles from Digital Ecosystems, and sustainability from Green 
Computing, while remaining true to the original vision of the Internet.

\section{RELATED WORK}

Dong etal. (2013) [30] has studied that as with the progress of cloud computing, many applications have been reinforced to offer in the cloud computing, which in turn increase the availability. As the resources are for the most part scattered, dynamic and heterogeneous so first and foremost, a checking model of distributed computing resources accessibility is made and after that, as indicated by the element procedure of the distributed computing administration, the accessibility of distributed computing resources is explored from Qos of a solitary cloud resources hub which is characterized by common attribution and unique attribution to Qos of some cloud resources which are associated by arrangement model, parallel model and blend model to give administration.

Meng et al. (2013) [31] has analyzed the cloud computing security in digital library. The distributed computing methodology of library computerized assets is characterized, then a social events of databases and system assets is received to give their administration, then these assets and offices are put in the cloud. And after that the cloud key dissemination plan to select to library applications is carried out. The better conventional PKI, the PKI-based distributed computing correspondence and mystery assurance components for library are presented.

Chalse et al. (2013) [32] has analysed the cloud security problem, the various issues in a cloud computing system and their effect upon the different cloud users. As the distributed computing environment is built focused around open Architecture and interface so the different registering framework and their result upon the framework, upon associations furthermore upon distinctive cloud administrators are broke down. A perspective of exercises that might be brought to manage the cloud security issue and counteractive action that must be considered by any association and cloud clients looking for venture in distributed computing are likewise introduced.

Jaber et al. (2013) [33] has demonstrated that the cloud computing i.e. the cloud computing is gaining popularity in every field. Likewise the distributed computing launched from a business endeavor idea, and made into a solid IT creation. Truth be told the distributed computing is ahead at the same time, clients stay reluctant to compose their business undertaking into the cloud which is because of the absence of assurance. The primary reason is the multifaceted nature which is included in dealing with the data on the cloud. So the distinctive cryptography perspectives that cause a risk to the distributed computing environment ought to be known to the client.

Guan et al. (2013) [34] has discussed the gaining popularity of cloud computing and the various problems associated with the hardware and software faults and the various factors such as environmental. To recognize abnormal cloud practices, the cloud execution is observed and runtime execution information is gathered. The gathered information comprises of execution measurements for diverse sorts of disappointments, which show distinctive associations with the execution measurements. This paper has proposed the system that runs across the segments of diverse disappointment sorts in distributed computing bases. It additionally examined the execution metric by analyzing the systems to achieve proficient distinguishing proof.
Zhang et al. (2013) [35] has discussed that the Cloud computing as typical Internet-based applications is lacking in carrier-grade signalling control mechanism and cannot guarantee Quality of Service (QoS), which is in turn a barrier for telecom operator. On the other side, as the center indicating structural planning of Next Generation Networking (NGN), IP Multimedia Subsystem (IMS) is confronting the trouble of the nonappearance of creative worth included administrations. This paper has proposedan building design to procurement distributed computing administrations over IMS The cloud administrations are considered as the normal IMS applications and after that cloud clients are permitted to get to cloud benefits under the control of Session Initiation Protocol (SIP) indicating and Qos component of IMS.

Guan et al. (2012) [36] has discussed the gaining popularity of cloud computing and the various problems associated with the hardware and software faults and the various factors such as environmental. Dependability guarantee is critical for constructing justifiable cloud computing services. As the virtualization is an enabling technology for the cloud, it is important to explore the effect of virtualization on the cloud dependability. The cloud dependability analysis (CDA) framework with methods to describe failure behaviour in cloud computing infrastructures is proposed. The failuremetric DAGs (directed a cyclic graph) to examine the relationship of several performance metrics with failure actions in virtualized and non-virtualized systems is also proposed. The generated DAGs in the two environments are compared to examine the impact of virtualization on the cloud dependability.

Liu, W. (2012) [37] has studied the cloud computing. The distributed computing is identified with the other figuring's, for example, lattice registering, circulated processing, parallel processing etc. As the distributed computing is identified with the information stockpiling, dependability and administrations so the security is the primary concern of the distributed computing. The security can turn away the quick development of distributed computing. This paper talks about the few distributed computing frameworks and explores distributed computing security issues and its arrangements as indicated by the distributed computing ideas and characters. As the single security strategy can't resolve the distributed computing security issue such a variety of customary and new advances and systems ought to be utilized as a part of an aggregate way for ensuring the aggregate distributed computing framework.

Zhao, Wei, et al. (2012) [38] has discussed that the cloud computing delivers computing resources as a service over a web. As it is the rising innovation, it gets to be vital to assess the execution and security issues that distributed computing meets. Right now, showing and impersonation innovation has turned into a powerful apparatus in distributed computing examination gathering to handle these issues. This paper states that there are two sorts of distributed computing test systems, that is, test systems simply focused around programming and test systems focused around both programming and equipment.

Xin et al. (2012) [39] has studied the concept of cloud computing and stated that it is considered to be the next generation of information technology frameworkIt can convey dynamic asset pools, virtualization and high attainable quality. The new improvement accompanies loads of new difficulties also.as a come about a protected registering environment ought to be fabricated. The technique to make secure distributed computing is the multi-measurement structural planning. It is a three layer model at the first the client 
verification is key to affirm that client information can't be altered.

Mollah et al. (2012) [40] has studied the cloud computing which is one of the evolving topic in the field of information technology. It concentrate on all calculation assets and oversee mechanically through the product without obstruction. As there are diverse layers show in the distributed computing structural planning, administration sorts and so on subsequently the building design, preferences, stages, issues and difficulties, and so on. are concentrated on. The four eras of processing, for example, centralized server based figuring, individualized computing, customer server based registering and web server based processing individually has its own particular advantages and disadvantages. Consequently the distributed computing is the up and coming era of the figuring administrations.

Sim and Mong (2012) [41] examined the Agent-based cloud computing which is related with the design and development of software agents for enhancing cloud service discovery, service etc. An operator based model for developing programming devices and test couches for cloud asset administration. Executor based distributed computing presents the creating of Cloudle: an operator based web crawler for cloud administration identification, demonstrating that operator based co-operation instruments could be productively acknowledged for boosting cloud administration co-operation and cloud business, and displaying that executor based agreeable critical thinking procedures might be viably received for robotizing cloud administration creation. For the cloud business, this created a multifaceted cloud arrangement component that backings parallel transaction exercises in interrelated markets: a cloud administration advertise between customer executors and merchant operators, and various cloud asset advertises between dealer operators and supplier operators.

\section{GAPS IN EARLIER WORK}

The review has shown that the most of the existing research has neglected most of the followings things:-

a. The use of scalability in data centers has been neglected by many researchers.

b. The failures in the data centers has been neglected in the most of the existing research.

c. The failover issues has been ignored by the most of the researchers.

\section{CONCLUSION}

Failures in the data centers has been ignored by the most of the existing research. It has been found that the most of the existing researchers has neglected many issues. Scalability, Failures and Failover in cloud data centers has been ignored in the most of the existing research. So in near future, the various techniques which can be used to overcome the data centers will be used. Also the use of the "secondary sleep mode high end servers" and also the use of "secondary data links" will be introduced in near future to overcome the failures.

\section{REFERENCES}

[1] Dong, Wang En, Wu Nan, and Li Xu. "QoS-Oriented Monitoring Model of Cloud Computing Resources Availability." Computational and Information Sciences (ICCIS), 2013 Fifth International Conference on. IEEE, 2013.
[2] Meng, Qingjie, and Changqing Gong. "Research of cloud computing security in digital library." Information Management, Innovation Management and Industrial Engineering (ICIII), 2013 6th International Conference on. Vol. 2. IEEE, 2013.

[3] Chalse, Rajkumar, Ashwin Selokar, and Arun Katara. "A New Technique of Data Integrity for Analysis of the Cloud Computing Security." Computational Intelligence and Communication Networks (CICN), 2013 5th International Conference on. IEEE, 2013.

[4] Eric , Galen Gruman, "What cloud computing really means," 19 Jul 2010, http://www.infoworld.com/d/cloud-computing.html

[5] Daniel J. Abadi, "Data Management in the Cloud: Limitations and Opportunities," Jul 2010 http://citeseerx.ist.psu.edu/viewdoc/download?rep=rep1 \&type $=$ pdf

[6] Dr. Wendy, A. Warr and Wendy Warr," Cloud computing," 29 Nov 2009, www.qsarworld.com/files/Cloud-computing.pdf

[7] Ivailo P. Sokolov, "Cloud Computing: Overview, Concepts and Business Deployment Scenarios," Vienna University of Economics and Business,2009, www.ivosokolov.com/papers/Cloud_Computing_Ivo_So kolov.pdf

[8] Jaakko Jäätmaa ,"Financial Aspects of Cloud Computing Business Models," Aalto university,2010 hsepubl.lib.hse.fi/FI/epaper/pdf/12435/hse_epaper_1243 5.pdf

[9] Victor Delgado,"Exploring the limits of cloud computing," Kungliga Tekniska Högskolan (KTH), Stockholm, Sweden, 4 Oct 2010 , www.ict.kth.se/ maguire./101118-Victor_Delgado-withcover.pdf

[10] Modine, "Web startups crumble under Amazon S3 outage," The Register, Tech. Rep., 2008. [Online]. Available: http://www. theregister.co.uk/2008/02/15/amazon s3 outage feb 2008/

[11] J. Montgomery, "Google Apps sees 99.9reliability"," Tech.Blorge, Tech. Rep., 2008. [Online]. Available: http://tech.blorge.com/Structure:\%20/2008/11/02/googleapps-sees-999-uptime-proves-cloud-reliability/

[12] J. Perez, "Google Apps customers miffed over downtime," IDG News Service, Tech. Rep., 2007. [Online]. Available http://www.pcworld.com/businesscenter/article/130234/g oogle apps customers miffed over downtime.html

[13] Kable, "Carter recommends 'g cloud' for gov it," The Register,2009. [Online]. Available: http://www.channelregister.co.uk/2009/06/17/governmen t cloud computing/

[14] J. Hayes, "Cred - or croak?" IET Knowledge Network, Tech.Rep., 2008. [Online]. Available: http://kn.theiet.org/magazine/issues/0820/cred-croak0820.cfm?SaveToPDF

[15] J. Kaplan,W. Forrest, and N. Kindler, "Revolutionizing data center energy efficiency," McKinsey \& Company, Tech. Rep., 2008. [Online]. Available: 
http://www.mckinsey.com/clientservice/bto/pointofview/ pdf/Revolutionizing Data Center Efficiency.pdf

[16] P. Mckenna, "Can we stop the internet destroying our planet?"New Scientist, vol. 197, no. 2637, pp. 20-21, 2008.

[17] Environmental Protection Agency, "EPA report to congress onserver and data center energy efficiency," US Congress, Tech.Rep., 2007.

[18] R. Miller, "NSA maxes out Baltimore power grid," Data Center Knowledge, Tech. Rep., 2006. [Online]. Available:

http://www.datacenterknowledge.com/archives/2006/08/ 06/nsa-maxes-out-baltimore-power-grid/

[19] K. McIsaac, "The data centre goes green, the CFO saves money,'Intelligent Business Research Services, Tech. Rep., 2007.

[20] R. Talaber, T. Brey, and L. Lamers, "Using virtualization to improve data center efficiency," The Green Grid, Tech. Rep., 2009.

[21] K. Brill, "The invisible crisis in the data center: The economic meltdown of Moore's law," Uptime Institute, Tech. Rep., 2007.

[22] J. Brodkin, "Gartner in 'green' data centre warning," Techworld,2008. [Online]. Available: http://www.techworld.com/greenit/news/index.cfm?newsid=106292

[23] Google, "Google App Engine: Run your web apps on Google's infrastructure." Google, Tech. Rep., 2009. [Online]. Available: http://code.google.com/appengine/

[24] Amazon, "Amazon Elastic Compute Cloud (EC2)," Amazon Web Services LLC, Tech. Rep., 2009. [Online]. Available: http://aws.amazon.com/ec2/

[25] Microsoft, "Azure services platform," Micrsoft, Tech. Rep., 2009.[Online]. Available: http://www.microsoft.com/azure/

[26] C . Metz, "The Meta Cloud - flying data centers enter fourth dimension," The Register, Tech. Rep., 2009. [Online]. Available: http://www.theregister.co.uk/2009/02/24/the meta cloud/

[27] C.Metz, "The Meta Cloud - flying data centers enter fourth dimension," The Register, Tech. Rep., 2009. [Online]. Available: http://www.theregister.co.uk/2009/02/24/the meta cloud/

[28] D. Nurmi, R. Wolski, C. Grzegorczyk, G. Obertelli, S. Soman, L. Youseff, and D. Zagorodnov, "The Eucalyptus open-source cloud-computing system," in Cloud Computing and Its Applications, 2008.

[29] M. Haynie, "Enterprise cloud services: Deriving business value from Cloud Computing," Micro Focus, Tech. Rep., 2009.

[30] Dong, Wang En, Wu Nan, and Li Xu. "QoS-Oriented Monitoring Model of Cloud Computing Resources
Availability." Computational and Information Sciences (ICCIS), 2013 Fifth International Conference on. IEEE, 2013.

[31] Meng, Qingjie, and Changqing Gong. "Research of cloud computing security in digital library." Information Management, Innovation Management and Industrial Engineering (ICIII), 2013 6th International Conference on. Vol. 2. IEEE, 2013.

[32] Chalse, Rajkumar, AshwinSelokar, and ArunKatara. "A New Technique of Data Integrity for Analysis of the Cloud Computing Security." Computational Intelligence and Communication Networks (CICN), 2013 5th International Conference on. IEEE, 2013.

[33] Jaber, AwsNaser, and MohamadFadli Bin Zolkipli. "Use of cryptography in cloud computing." Control System, Computing and Engineering (ICCSCE), 2013 IEEE International Conference on. IEEE, 2013.

[34] Guan, Qiang, and Song Fu. "Adaptive Anomaly Identification by Exploring Metric Subspace in Cloud Computing Infrastructures." Reliable Distributed Systems (SRDS), 2013 IEEE 32nd International Symposium on. IEEE, 2013

[35] Zhang, W., Lei, W., Chen, X., \& Liu, S. (2013, June) Architecture and Key Issues of IMS-Based Cloud Computing. In Cloud Computing (CLOUD), 2013 IEEE Sixth International Conference on (pp. 629-635). IEEE.

[36] Guan, Qiang, Chi-Chen Chiu, and Song Fu. "CDA: A Cloud Dependability Analysis Framework for Characterizing System Dependability in Cloud Computing Infrastructures." Dependable Computing (PRDC), 2012 IEEE 18th Pacific Rim International Symposium on. IEEE, 2012

[37] Liu, Wentao. "Research on cloud computing security problem and strategy."Consumer Electronics, Communications and Networks (CECNet), 2012 2nd International Conference on. IEEE, 2012.

[38] Zhao, Wei, et al. "Modeling and simulation of cloud computing: A review." Cloud Computing Congress (APCloudCC), 2012 IEEE Asia Pacific. IEEE, 2012.

[39] Xin, Zhang, Lai Song-qing, and Liu Nai-wen. "Research on cloud computing data security model based on multidimension." Information Technology in Medicine and Education (ITME), 2012 International Symposium on. Vol. 2. IEEE, 2012.

[40] Mollah, Muhammad Baqer, KaziReazul Islam, and Sikder Sunbeam Islam. "Next generation of computing through cloud computing technology." Electrical \& Computer Engineering (CCECE), 2012 25th IEEE Canadian Conference on. IEEE, 2012.

[41] Sim, Kwang Mong. "Agent-based cloud computing." Services Computing, IEEE Transactions on 5.4 (2012): 564-577. 